\title{
Guanylate cyclase $C$ signaling: an intestinal secretory pathway where bugs, genes and new drugs intersect
}

\author{
Michael Camilleri*
}

\begin{abstract}
Acute infectious and chronic diarrheal diseases are important public health problems. A recent study by Fiskerstrand and colleagues identified a family with a rare early onset familial diarrhea. By linkage analysis and exon sequencing, the authors identified a heterozygous missense mutation in GUCY2C, encoding the guanylate cyclase $C$ receptor, which is involved in intestinal secretion. This newly identified gene in the etiology of a familial diarrhea provides a candidate target for the development not only of new treatments for diarrhea, but also of a new drug class to treat constipation.

Keywords Agonist, diarrhea, familial, genetic, guanylate cyclase C, GUCY2C, secretion.
\end{abstract}

\section{Intestinal secretory mechanisms and diarrheal diseases}

Diarrhea, caused by acute infectious or chronic diseases, may be considered as an imbalance between the approximately $7 \mathrm{l}$ of fluids ingested or secreted into the gastrointestinal tract each day, and the reabsorption of almost an equivalent volume. Such an imbalance may lead to malabsorption of nutrients, dehydration and electrolyte imbalances. Infectious organisms such as Escherichia coli or Vibrio cholerae produce toxins that stimulate normal secretory pathways in the intestine, resulting in potentially life-threatening secretory diarrhea and dehydration. The gold standard of treatment is rehydration with glucose-electrolyte solution but, regrettably, there is still

*Correspondence: camilleri.michael@mayo.edu

Clinical Enteric Neuroscience Translational and Epidemiological Research

(C.E.N.T.E.R.), College of Medicine, Mayo Clinic, Charlton 8-110, 200 First Street SW, Rochester, MN 55905, USA a high mortality from these conditions in developing countries; hence, more effective treatments are needed.

Intestinal secretion may result from stimulation of a number of secretory pathways. For example, $V$. cholerae infection induces enteroendocrine cells to secrete 5-hydroxytryptamine (5-HT or serotonin), which stimulates secretion of water and electrolytes from small intestinal epithelial cells or enterocytes [1]. In addition, cholera is associated with outpouring of mucin from goblet cells. It has been shown that secretions from goblet cells include the peptides guanylin [2] and uroguanylin, which have similar amino acid sequences to the heat-stable enterotoxin of E. coli. These enterotoxins and endogenous peptides bind to guanylate cyclase $\mathrm{C}$ (GC-C) and stimulate the production of increased levels of intracellular cyclic guanosine monophosphate (cGMP). Increased levels of cGMP ultimately activate the secretion of chloride ions through the cystic fibrosis transmembrane conductance regulator (CFTR). When chloride reaches the intestinal lumen, its osmotic force pulls water out of the interstitial fluid through the spaces between enterocytes and this ultimately leads to dehydration.

It has been demonstrated that enterotoxigenic E. coli requires the GC-C receptor to induce intestinal secretion [3]. In addition to its presence in goblet cells, guanylin is also located in enteroendocrine cells, as well as in neuroendocrine tumors such as carcinoids. In fact, enhanced local secretion of guanylin may contribute to secretory diarrhea in these patients [4]. The relationship of enterocytes, goblet cells and enteroendocrine cells in the lining of the intestine and the role of secretomotor mechanisms has been reviewed previously [5]. Although some of the signaling pathways involved in abnormal intestinal secretion have been identified, targeted approaches to reverse secretion are needed to complement rehydration therapy. This article highlights a recent study that identifies the molecular genetic basis of a familial diarrhea (FD), illustrating a mechanism that can be pharmacologically targeted to enhance secretion in constipation or to inhibit secretion in diarrheal diseases. 


\section{Diarrhea due to mutation in GUCY2C}

Fiskerstrand et al. [6] identified a Norwegian family with a rare, early onset form of chronic or intermittent FD. Their investigation started with careful clinical observations of an 88-year-old admitted to hospital for acute diarrhea and dehydration. It was noted that affected members of the family started to develop symptoms in infancy, had chronic, relatively mild diarrhea, and the symptoms tended to subside by middle age in some members of the family. The FD was a dominantly inherited, fully penetrant disease in 32 (18 male and 14 female) members of the family. Fourteen unaffected members of the family were also evaluated.

In a series of elegant studies, the authors used whole genome linkage analysis based on arrays with SNPs to identify a candidate region on chromosome 12 , and then sequenced GUCY2C, encoding GC-C. Linkage analysis was used to determine the candidate region for the mutation in samples from 11 affected members and 14 unaffected members. This analysis revealed only one shared region in the affected members, located on the short arm of chromosome 12 (12p) with a maximum LOD (logarithm (base 10) of odds) score of 5.1. A haplotype spanning approximately 2.9 megabases showed complete segregation with the disease, and the region contained 28 putative protein coding genes, including GUCY2C. Sequence analysis identified a heterozygous base substitution, c.2519G $\rightarrow$ T, in exon 22. This SNP was identified in all affected family members. Exome sequencing of the entire candidate region from three affected family members excluded mutations in genes other than GUCY2C as possible causes of or contributors to susceptibility to the disease.

In addition, Fiskerstrand et al. [6] conducted functional studies of mutant GC-C in HEK293T cells. Exposure of the mutant receptor to its ligands (heat-stable enterotoxin, uroguanylin and guanylin) resulted in markedly increased (up to eightfold) production of cGMP. These functional assays demonstrate that the mutation is capable of inducing the intracellular production of the second messenger cGMP, which results in intestinal secretion through CFTR.

The authors appropriately describe this familial variation as a form of 'genetic knock-in' of the mechanism that is stimulated by enterotoxigenic E. coli, and this represents an intriguing confluence of the effects of bugs and genes.

\section{Clinical significance}

The Fiskerstrand et al. study [6], which identified the causative GUCY2C mutation, has important implications from mechanistic, diagnostic and therapeutic perspectives. First, identification of this gene mutation in FD provides evidence that the pathway is an important mechanism of chronic diarrhea in addition to infectious enterotoxic diarrhea. Second, mutation in GUCY2C should be considered in the differential diagnosis of chronic diarrhea, especially when there is a positive history of FD. Third, understanding the pathway provides a target for the development of a new drug class to treat constipation by inducing intestinal secretion. Since analogs of guanylin and uroguanylin (linaclotide and plecanatide $[7,8]$ ) already exist, clinical translation of the science to develop secretagogue drugs for relief of constipation and associated symptoms (bloating and abdominal pain) had preceded the intriguing molecular genetic discovery of Fiskerstrand et al. [6]. Targeting the receptor represents an alternative therapeutic approach, in lieu of targeting the gene itself.

However, there are some loose ends in the story. The susceptibility of the Norwegian family to inflammatory bowel disease (IBD), small bowel obstruction and esophagitis in the absence of hiatal hernia cannot be easily attributed to the effects of the GUCY2C mutation on GC-C, based on current knowledge. In addition, there are no reports of any association of the GUCY2C mutation and IBD, even with genome-wide association studies $[9,10]$. Nevertheless, the GC-C agonists plecanatide and SP-333 (analogs of uroguanylin) have been shown to ameliorate inflammation in murine colitis models via downregulation of certain pro-inflammatory cytokines, such as IL-6, IL-17 and IL-23 [9], and they may also have therapeutic potential in IBD and, possibly, carcinogenesis [8].

This remarkable story may eventually have a happy ending for patients with this specific FD. The report should stimulate the development of blockers of the GC$\mathrm{C}$ and CFTR intestinal secretory pathways, and this will have potential for wide application to life-threatening infectious diarrheas, in addition to the chronic, mild, non-fatal diarrhea afflicting this family; these diarrheas could then be specifically targeted with personalized or precision medicine.

\section{Abbreviations}

CGMP, cyclic guanosine monophosphate; CFTR, cystic fibrosis transmembrane conductance regulator; FD, familial diarrhea; GC-C, guanylate cyclase C; $\mathrm{IBD}$, inflammatory bowel disease; IL, interleukin; SNP, single-nucleotide polymorphism.

Competing interests

The author declares that he has no competing interests.

Published: 26 June 2012

References

1. Nilsson O, Cassuto J, Larsson PA, Jodal M, Lidberg P, Ahlman H, Dahlström A, Lundgren O: 5-hydroxytryptamine and cholera secretion: a histochemical and physiological study in cats. Gut 1983, 24:542-548.

2. Cohen MB, Witte DP, Hawkins JA, Currie MG: Immunohistochemical localization of guanylin in the rat small intestine and colon. Biochem Biophys Res Commun 1995, 209:803-808.

3. Charney AN, Eqnor RW, Alexander-Chacko JT, Zaharia V, Mann EA, Giannella 
RA: Effect of $E$. coli heat-stable enterotoxin on colonic transport in guanylyl cyclase C receptor-deficient mice. Am J Physio/ 2001, 280:G216-G221.

4. Kuhn M, Kulaksiz H, Cetin Y, Frank M, Nold R, Arnold R, Böker K, Bischoff SC, Manns MP, Forssmann WG: Circulating and tissue guanylin

immunoreactivity in intestinal secretory diarrhoea. Eur J Clin Invest 1995, 25:899-905.

5. Camilleri M, Nullens S, Nelsen T: Enteroendocrine and neuronal mechanisms in pathophysiology of acute infectious diarrhea. Dig Dis Sci 2012, 57:19-27.

6. Fiskerstrand T, Arshad N, Haukanes BI, Tronstad RR, Pham KD, Johansson S, Håvik B, Tønder SL, Levy SE, Brackman D, Boman H, Biswas KH, Apold J, Hovdenak N, Visweswariah SS, Knappskog PM: Familial diarrhea syndrome caused by an activating GUCY2C mutation. N Engl J Med 2012,

366:1586-1595

7. Lembo AJ, Schneier HA, Shiff SJ, Kurtz CB, MacDougall JE, Jia XD, Shao JZ, Lavins BJ, Currie MG, Fitch DA, Jeglinski Bl, Eng P, Fox SM, Johnston JM: Two randomized trials of linaclotide for chronic constipation. N Engl J Med 2011 , 365:527-536.
8. Shailubhai K, Nefsky B, Masih S, Foss J, Comiskey SC, Jacob GS, Plevy SE: Guanylate cyclase $C$ agonists, a new class of drug candidates for treatment of inflammatory bowel disease. Am J Gastroenterol 2011, 106:S455, 1208.

9. Lee JC, Parkes M: Genome-wide association studies and Crohn's disease. Brief Funct Genomics 2011, 10:71-76.

10. Thompson Al, Lees CW: Genetics of ulcerative colitis. Inflamm Bowel Dis $2011,17: 831-848$.

doi:10.1186/gm349

Cite this article as: Camilleri M: Guanylate cyclase C signaling: an intestinal secretory pathway where bugs, genes and new drugs intersect. Genome Medicine 2012, 4:50 